\title{
El profesorado y su percepción sobre la igualdad de género en la universidad ${ }^{1}$
}

\section{Teaching staff and their perception about gender equality at university}

\author{
Georgeta ION, Maria del Mar DURAN-BELLONCH \\ y Maria Dolors BERNABEU TAMAYO \\ Universidad Autónoma de Barcelona
}

Recibido: Septiembre 2012

Aceptado: Noviembre 2012

\section{Resumen}

En este artículo se cuestiona si actualmente podemos seguir hablando de una situación de inequidad de género en las universidades. Desde la revisión bibliográfica se puede decir que a pesar de que en las universidades se han tomado medidas en los últimos años para fomentar la igualdad de género en sus estructuras y prácticas, todavía existe desigualdad, en tanto que se constata la escasa presencia femenina en algunos de los ámbitos de la vida universitaria de nuestro país.

La principal aportación del artículo la constituyen los resultados derivados de una investigación realizada en dos universidades españolas, utilizando metodología cualitativa, a fin de ahondar en las percepciones de las personas académicas en relación a la igualdad de género. Se llevan a cabo 22 entrevistas en profundidad.

Los resultados de la investigación señalan que sigue existiendo inequidad y ofrecen datos más concretos sobre los posibles motivos que la perpetúan. Por un lado aquellos relativos a lo personal y lo organizativo. Uno de ellos la necesidad de conciliar vida profesional y familiar. La atención a la familia sigue estando asociada al género femenino. A pesar de la mayor implicación masculina, para los hombres todavía el trabajo familiar no les supone un esfuerzo comparable al que supone a las mujeres, que siguen llevando la mayor parte de la carga de trabajo, más allá de la maternidad, lo que condiciona directamente que puedan obtener los mismos méritos que los hombres durante su carrera académica. Otro motivo de desigualdad se halla también en la cultura organizativa de las universidades españolas, que ha sido y sigue

${ }^{1}$ El articulo forma parte de la investigación "Estudio de las dimensiones de visibilidad de las profesoras en la universidad contemporánea", financiada por el Ministerio de Trabajo y Asuntos Sociales (ref. 005/007- coordinadora Dra. Marina Tomàs). 
siendo muy masculinizada y tiende a conllevar la exclusión femenina. Por otro lado, las políticas de igualdad, que no son percibidas como suficientemente satisfactorias por las personas entrevistadas. Los resultados de la investigación indican también la falta de equidad de género entre el personal docente universitario, sobre todo en lo relacionado a la función investigadora y gestora y de su perfil profesional.

Palabras clave: género, políticas de género, carrera académica, equidad, igualdad sexos.

\begin{abstract}
The article makes important contributions in order to clarify if we can continue talking about a situation of gender inequality in the universities. We performed a systematic literature review, which includes from pioneers in the field to the more recent work. From this review it appears that even though universities have taken steps in recent years to promote gender equality in their structures and practices, there is still inequality, in that it confirms the low female presence in some of the areas of university life in our country. The second contribution the results are derived from research conducted in two Spanish universities, using qualitative methodology in order to delve into the perceptions of academic people in relation to gender equality. The methodology is qualitative and is based on 22 interviews. The participants are academics from two Spanish public universities.

The need to balance work and family life, which is still a need associated with female gender, despite the greater involvement of men in family responsibilities, for men still the family responsibilities don not requires an effort comparable to the women involved, that still bears the bulk of the workload, beyond motherhood, which directly determines who can get the same merit than men during their academic career. Also the organizational culture of our universities, which has been and remains very masculine, is a reason for female exclusion.

Finally, our results of our investigation warn about the lack of gender equity in different academic fields, especially present in research and management.
\end{abstract}

Keywords: gender, gender policy, academic career, equity.

En las últimas décadas el concepto de equidad ha sido ampliamente estudiado, también en el ámbito académico universitario. Hace referencia a diversos aspectos como el género, la etnia, las necesidades especiales, la orientación sexual, la edad, la clase social, el nivel profesional, el tipo de trabajo realizado, el nivel educativo alcanzado, la apariencia física u otros aspectos que pueden ser causa de discriminación o de desigualdad. De todos estos aspectos, en el presente artículo nos centramos exclusivamente en aquellos que afectan a la igualdad de género entre el profesorado universitario.

A pesar de que la universidad se define socialmente como un campo para la igualdad de oportunidades en base a un sistema meritocrático de funcionamiento (Alonso, 2001,73) y de que los agentes implicados han interiorizado los objetivos y las expectativas profesionales de dicho sistema meritocrático, "la estructura social típica del campo universitario se conforma a partir de un mercado de trabajo interno, caracterizado por la exclusión de las mujeres de las posiciones de poder y prestigio. La inequidad de género es uno de los aspectos que afectan las relaciones sociales en casi todas las sociedades. Estructura las relaciones de producción y reproducción del 
conocimiento y está directamente ligada a su construcción y difusión" (Alonso, 2001,73).

Las estadísticas en España son contundentes (Morales, Luna y Esteban 2010) y demuestran la poca presencia femenina en algunos de los ámbitos de la vida universitaria. Las cifras no son muy diferentes en el resto de países europeos, e incluso aquellos que desarrollan sofisticadas políticas de igualdad siguen teniendo un bajo número de mujeres ocupando posiciones de alto nivel en docencia, investigación o gestión. Además, la equidad entre hombres y mujeres no se reduce solamente a un aspecto cuantitativo, sino que implica actuaciones sutiles de índole cualitativa. Por ello se puede afirmar que son necesarios cambios micro-políticos que hagan que la academia sea un lugar más inclusivo. En este proceso podemos preguntarnos: ¿qué es lo que se debería igualar cuando nos proponemos alcanzar la equidad o qué aspectos de la inequidad de género son los que provocan más malestar en la universidad?

En este artículo nos proponemos en la primera parte contribuir a delimitar el concepto de igualdad de género y ofrecer una síntesis del estado actual de la investigación sobre el tema, concretamente en el ámbito universitario. En la segunda parte, después de hacer una breve mención a la metodología utilizada en nuestra investigación, exponemos algunos resultados de la misma, concretamente aquellos que tienen que ver directamente con el sentir de las personas participantes en relación a la igualdad de género en la universidad a inicios de la segunda década del siglo XXI.

\section{Sobre la igualdad de género}

A finales de los años noventa, los gobiernos de distintas naciones tomaron varias iniciativas para promover la igualdad, todavía no conseguida, entre los hombres y las mujeres. En el ámbito científico, autores como Rees (1998), proponían un enfoque para asegurar la igualdad de género, denominado "acción positiva". Ello significaba la adopción de medidas dirigidas hacia las mujeres para mejorar su posición en una sociedad patriarcal. "La acción positiva, tiene como extremo la discriminación positiva que apunta hacia el fomento de la participación de las mujeres (o de otros grupos minoritarios) a través de acciones afirmativas preferentes o cuotas" (Rees 1998, 37). Junto a estas medidas, otras más prometedoras son las que Rees (1998) identifica bajo el concepto de 'gender mainstreaming'. El concepto comprende una serie de iniciativas destinadas a la incorporación sistemática de los aspectos de género en todas las instituciones y en las políticas públicas. Tal como lo define la Comisión Europea, es un concepto que implica un compromiso de integración sistemática de las situaciones, prioridades y necesidades de los hombres y de las mujeres en todas las políticas, con el propósito de promover la igualdad entre hombres y mujeres movilizando todos los recursos adecuados para dar respuesta a este objetivo (Commission of the European Communities, 1996). 


\section{La igualdad de género en la educación superior}

También se han dedicado esfuerzos a analizar científicamente aspectos relacionados con la igualdad de género en la universidad. Son una muestra los trabajos de Bagilhole (2002), David (2003), Howie y Tauchert (2002), Leonard (2001), Morley (2005) que relacionan el género con las desigualdades sociales en los países del Commonwealth, Deem y Ozga (2000) que analizan los temas de equidad poniendo de manifiesto que cuando esta existe se relaciona más con estudiantes que con docentes, Søyland, y otros (2000); Harley (2003) y Husu (2000) en el contexto europeo. Estos dos últimos autores, se han dedicado a analizar las políticas implementadas al nivel europeo haciendo énfasis en que a pesar de la multitud de medidas, las mujeres siguen estando poco representadas en los puestos de poder. Los trabajos de Blackmore y Sachs (2001), Chesterman (2002), y Probert, B., Ewer, P. \& Whiting, K. (1998), en Australia examinan las complexidades de los obstáculos estructurales, actitudinales y psicológicas de la equidad de género en las organizaciones patriarcales. . En Canadá los estudios de Acker (1996). En Nueva Zelanda los de Brooks (1997). En Sud-África los de De La Rey (2001) y en Singapur, Hong Kong y Tailandia los de Luke (2001).

Los temas centrales de las investigaciones coinciden y constatan el mismo problema: la escasa presencia de las mujeres en diversos ámbitos académicos, especialmente aquellos directamente vinculados con la investigación y con los puestos de mayor responsabilidad y poder. Al inicio del siglo XX, además de constatar lo dicho, las y los investigadores se dedicaron, también, a estudiar qué políticas de igualdad se pusieron en marcha y cuáles fueron sus resultados iniciales.

Las metas que debían alcanzar los países europeos en relación a la igualdad en la educación superior, según el Informe Eurydice (2010), eran dos: acabar con la segregación horizontal y con la segregación vertical que, como indican los estudios realizados, continúan siendo una realidad en nuestro sistema universitario.

La segregación horizontal se refiere al hecho de que las mujeres y los hombres siguen eligiendo diferentes campos disciplinares en la educación superior, siendo en las ingenierías y en las ciencias experimentales dónde hay un menor número de mujeres. Kodate, N; Kodate, K y Kodate, T (2010), por ejemplo, constatan en su trabajo que dicha segregación sigue existiendo.

La segregación vertical es un problema relacionado con el llamado "techo de cristal", también presente en la educación superior. Mientras que las mujeres superan a los hombres entre los titulados superiores, son pocas las que cursan estudios de doctorado y menos aún las que pasan a formar parte del personal académico en las universidades. Las que consiguen acceder a la carrera académica se concentran en los niveles más bajos de la jerarquía, ocupando los puestos más inestables, muchas veces asociados con la docencia o solamente con la investigación cuando son 'ayudantes de' (Bryson \& Barnes, 2000). Otros estudios, como los de Lunn (2007) y los de Leathwood y Read (2008), corroboran el dato: el aumento de la presencia de profesoras e investigadoras en la universidad no corresponde a su participación real en el conocimiento científico y en las estructuras de poder en la universidad. 
En España, las proporciones se mantienen sin mucha variación y coinciden con las europeas. Ya Alberdi en 1996 decía: "El prestigio, los ingresos, el poder, el acceso a la gestión de las universidades sigue estando de forma abrumadoramente mayoritaria en manos masculinas. El género persiste como criterio diferenciador de poder en las universidades. Las razones pueden ser muchas, la realidad es indiscutible. La presencia de mujeres tiende a reducirse según se asciende de nivel, de categoría y de prestigio en la universidad." (Alberdi, 1996, 77). Un lustro después, las profesoras seguían estando en la misma situación de inferioridad, como muestran los estudios de Alonso (2001), entre otros. Desafortunadamente, estudios más recientes realizados en universidades españolas siguen mostrando la existencia de desigualdad entre géneros. Por ejemplo, siguen habiendo estereotipos de género entre estudiantes universitarios (Sánchez García y otros, 2011) o siguen dándose los fenómenos de desigualdad debido a las barreras que intervienen en la construcción de la carrera universitaria (Donoso, Figuera, y Rodríguez, 2011). De las aportaciones de Ion y Duran (2011) sobre los factores que contribuyen al éxito de las pocas investigadoras que consiguen destacar en sus respectivos ámbitos científicos, se desprenden las dificultades que ellas han tenido que superar para llegar dónde están en las universidades catalanas, en comparación con las trayectorias seguidas por sus colegas masculinos. Por todo ello, luchar por la igualdad de género en las universidades españolas sigue siendo una necesidad social perentoria. A ese fin, en los últimos tiempos se han tomado algunas medidas para mejorar la situación y se han generado políticas de cuotas que suponen cambios importantes en la composición de los departamentos, o de los comités de selección, entre otros, que están teniendo el efecto de ir equiparando en número a mujeres y hombres que ocupan cargos académicos.

Siguiendo con las acciones destinadas a conseguir la equidad entre profesores y profesoras, durante los últimos cinco años en muchas universidades se han creado los llamados 'Observatorios para la Igualdad de Género' que crean y gestionan actividades encaminadas a conseguir una universidad más justa (e.j. Primer Congreso Internacional sobre Sesgo de Género y Desigualdades en la Evaluación de la Calidad Académica, 2008, Jornada 'La Presencia de las Mujeres en los Cargos de Dirección, 2011).

En el contexto expuesto, se realizó la investigación que presentamos a continuación, sobre la percepción de desigualdades de género en la universidad y la eficacia de las estrategias iniciadas para superarlas.

\section{Método}

Para alcanzar nuestro objetivo, nos hemos situamos dentro del paradigma interpretativo y hemos utilizado la entrevista como técnica para conseguir un tipo de información que solo puede obtenerse a través de la relación 'cara a cara' que se establece entre dos personas cuando hablan de un tema. Se trata de establecer una relación interpersonal con el entrevistado que, siguiendo a Woods (1989) pueda descubrirse como éste entiende y vive un fenómeno determinado. Durante una entrevista pueden llegarse a detectar fachadas públicas e ir más allá para captar aquello esencial, el significado más íntimo que la persona otorga a una realidad dada. 
Las entrevistas han sido semiestructuradas, el equipo investigador partió de un guión temático para focalizar el tema a tratar. El guión estaba compuesto por preguntas abiertas sobre los principales ámbitos de actuación del profesorado universitario en relación al poder, a la participación y a las características de quien es considerado experto en su ámbito de conocimiento. En relación al tema de la equidad, se preguntaba si se creía que existía desigualdad entre géneros en la Universidad y en caso afirmativo en qué momento del desarrollo profesional se producía dicha desigualdad en su opinión. También se pedía a los entrevistados que expusieran su punto de vista en relación a los cargos de gestión en la universidad y el género, si consideraban que había diferencias entre hombres y mujeres para acceder a ellos y mantenerse y si opinaban que había algún tipo de cargo de gestión en el que hubiese más presencia femenina y el porqué. El último punto relevante sobre el tema de la equidad que se trataba con las personas entrevistadas era sobre el concepto de persona experta y género.

Las entrevistas las realizaron los mismos miembros del equipo investigador, tuvieron distinta duración en función de la cantidad de tiempo que cada persona participante quería dispensar a cada tema. La media puede situarse en las dos horas por conversación.

\section{Muestra}

Partiendo de un muestro teórico (Bryman, 2004), se optó por un criterio de selección "por conveniencia" del profesorado participante en el estudio, cumpliendo los siguientes criterios:

- Que todos las personas seleccionadas fuesen profesionales reconocidos en, al menos, una de las funciones del personal docente e investigador de las universidades públicas españolas (docente, investigadora y gestora) $\mathrm{O}$ bien respondieran a un perfil mixto (personas que destacan por ser reconocidas en las tres funciones mencionadas).

- Que provinieran de todos los ámbitos de conocimiento.

- Que se mantuviese el equilibrio de género entre los entrevistados.

La colaboración de los entrevistados fue voluntaria y todos ellos provenían de dos universidades públicas españolas: la Universidad de Sevilla (USE) y la Universidad Autónoma de Barcelona (UAB). En total participaron 22 personas.

La distribución de la muestra en relación a los criterios comentados se muestra a continuación en la tabla 1. 


\begin{tabular}{|c|c|c|c|c|c|c|c|c|c|c|c|c|}
\hline $\mathbf{N}^{\mathbf{o}}$ & \multicolumn{4}{|c|}{ Pérfil } & \multicolumn{2}{|c|}{ Género } & \multicolumn{4}{|c|}{ Ámbito conocimiento } & \multicolumn{2}{|c|}{ Universidad } \\
\hline $\mathrm{E}$ & Docente. & Investigador & Gestión. & Mixto & $\mathrm{H}$ & M. & $\begin{array}{l}\text { C. } \\
\text { Socia. }\end{array}$ & $\begin{array}{l}\text { C. } \\
\text { Técn }\end{array}$ & $\begin{array}{l}\text { C. } \\
\text { Salud. }\end{array}$ & $\begin{array}{l}\text { C. } \\
\text { Experim. }\end{array}$ & UAB & USE \\
\hline 1 & & & $\mathrm{X}$ & & $\mathrm{X}$ & & $\mathrm{X}$ & & & & $X$ & \\
\hline 2 & & $X$ & & & $\mathrm{X}$ & & & & $\mathrm{X}$ & & $\mathrm{X}$ & \\
\hline 3 & & & $X$ & & $\mathrm{X}$ & & & $X$ & & & $\mathrm{X}$ & \\
\hline 4 & & $\mathrm{X}$ & & & & $\mathrm{X}$ & $\mathrm{X}$ & & & & $\mathrm{X}$ & \\
\hline 5 & & & & $\mathrm{X}$ & & $\mathrm{X}$ & & $\mathrm{X}$ & & & $\mathrm{X}$ & \\
\hline 6 & & $\mathrm{X}$ & & & & $\mathrm{X}$ & & & & $\mathrm{X}$ & $\mathrm{X}$ & \\
\hline 7 & $\mathrm{X}$ & & & & $\mathrm{X}$ & & & $\mathrm{X}$ & & & $\mathrm{X}$ & \\
\hline 8 & & & & $\mathrm{X}$ & $\mathrm{X}$ & & & & $\mathrm{X}$ & & $\mathrm{X}$ & \\
\hline 9 & & & $\mathrm{X}$ & & & $\mathrm{X}$ & & & $\mathrm{X}$ & & $\mathrm{X}$ & \\
\hline 10 & $\mathrm{X}$ & & & & & $\mathrm{X}$ & $\mathrm{X}$ & & & & $\mathrm{X}$ & \\
\hline 11 & & & & $\mathrm{X}$ & $\mathrm{X}$ & & & & & $X$ & $X$ & \\
\hline 12 & $\mathrm{X}$ & & & & & $\mathrm{X}$ & $\mathrm{X}$ & & & & & $\mathrm{X}$ \\
\hline 13 & $\mathrm{X}$ & & & & & $\mathrm{X}$ & & & & $X$ & & $\mathrm{X}$ \\
\hline 14 & & $X$ & & & & $X$ & & & & $X$ & & $X$ \\
\hline 15 & & $X$ & & & $X$ & & & $X$ & & & & $X$ \\
\hline 16 & & & & $X$ & $X$ & & $X$ & & & & & $X$ \\
\hline 17 & & & $X$ & & & $\mathrm{X}$ & $\mathrm{X}$ & & & & & $X$ \\
\hline 18 & & & $X$ & & $X$ & & & & $X$ & & & $X$ \\
\hline 19 & & & & $X$ & & $X$ & $X$ & & & & & $X$ \\
\hline 20 & & & & $X$ & $X$ & & & & & $X$ & & $X$ \\
\hline 21 & $X$ & & & & $X$ & & & $X$ & & & & $X$ \\
\hline 22 & $\mathrm{X}$ & & & & & $X$ & & & $\mathrm{X}$ & & & $X$ \\
\hline
\end{tabular}

TABLA I. Distribución de los participantes en la investigación según los criterios establecidos

\section{Análisis de la información}

Después de transcribir cada entrevista y someter el contenido a validación por los participantes, se analizó la información utilizando el programa informático de análisis de datos cualitativos MAXQDA versión 2007. En el análisis se partió de un sistema de categorías de tipo temático, coincidentes con las categorías de análisis predefinidas que constituyeron el guión de entrevista. En la tabla II se muestran las categorías definidas junto a las subcategorías halladas. Por ejemplo, se había definido previamente la categoría "ser experto o experta" y se preguntó a las personas entrevistadas sobre el significado otorgado a dicha categorías. Los datos se analizaron buscando respuestas a esta pregunta y de dicho análisis se dedujeron un conjunto de subcategorías. En este caso: la percepción sobre el reconocimiento académico en forma de publicaciones y dirección de proyectos; la presencia en los medios de comunicación y la presencia en diversos ámbitos de la actividad académica: docencia, investigación, gestión. 


\begin{tabular}{|c|c|c|c|}
\hline $\begin{array}{l}\text { Categoría de } \\
\text { análisis }\end{array}$ & Ser experto/a & Tener Poder & Participar \\
\hline $\begin{array}{l}\text { Subcategorias } \\
\text { de análisis }\end{array}$ & $\begin{array}{l}\text {-Percepción sobre el } \\
\text { reconocimiento académico: } \\
\text { publicaciones, dirección de } \\
\text { proyectos. } \\
\text { - Presencia en los medios de } \\
\text { comunicación. } \\
\text { - Presencia en diversos } \\
\text { ámbitos de la actividad } \\
\text { académica: docencia, } \\
\text { investigación, gestión. }\end{array}$ & $\begin{array}{l}\text {-Expectativas en } \\
\text { relación con el } \\
\text { sistema de } \\
\text { promoción. } \\
\text {-Obstáculos para la } \\
\text { promoción. } \\
\text {-Distribución de } \\
\text { recursos. } \\
\text { - Tipos de poder. }\end{array}$ & $\begin{array}{l}\text {-Presencia y } \\
\text { proporción en } \\
\text { diferentes } \\
\text { escenarios. } \\
\text {-Percepción sobre } \\
\text { las causas de la falta } \\
\text { de participación. }\end{array}$ \\
\hline
\end{tabular}

TABLA II: Ejemplo de categorías y subcategorias establecidas durante el análisis

\section{Resultados y discusión}

Todavía, a inicios de la segunda década del siglo XXI, el profesorado participante en nuestra investigación percibe una situación de desigualdad de género persistente en sus lugares de trabajo. Las razones que sustentan dicha desigualdad son similares a las halladas por los estudios realizados durante la década de los noventa y con anterioridad.

\section{Percepción sobre el grado de equidad entre géneros en el ámbito universitario}

Partiendo del análisis de los datos, podemos agrupar los motivos que causan desigualdad entre los logros conseguidos por hombres y por mujeres en la tarea investigadora, en dos grandes bloques. El primero hace referencia a la historia y los factores socioculturales que la han acompañado y nos han llevado a tener una sociedad en la que no se ha pensado en la necesidad de conciliar la vida profesional y la personal. Dicen nuestras fuentes:

"Socialmente [las mujeres] se han ocupado de otro tipo de actividades que están poco reconocidas, como la maternidad. Y este tipo de paradas [en la carrera académica] no se reconocen"; "La investigación, la propia formación, las publicaciones... no es lo mismo con cargas que no cargas. Y aunque los hombres también podemos decir que tenemos cargas, no es lo mismo [...]. Si ya cuesta a lo masculino, imagínate a lo femenino mucho más. Hay gente que incluso decide renunciar: no merece la pena hacer una apuesta tan arriesgada y tan importante sacrificando tanto a la familia" (E14). 
Nuestros resultados coinciden con los obtenidos en otras investigaciones (Evans, 2007; Drago y otros, 2006 y Gault \& Lovell 2006): muchas mujeres sienten que han de hacer frente a las expectativas sociales y esto las "sanciona" a nivel profesional. Aunque los hombres también pueden decidir formar una familia, a ellos este hecho no les condiciona su trabajo y menos su dedicación profesional. Todo lo contrario, la expectativa social en su caso es que han de ascender con más empeño, pues tienen una familia que mantener. Esto forma parte del inconsciente social y está tan interiorizado que aislarlo para cuestionárselo, para analizarlo y comprender la discriminación entre ambos géneros, no es algo sencillo (Andreu, 2002, 24). Sobre este tipo de fenómeno, una entrevistada explica:

"Yo quiero creer que la evaluación se aplica de igual modo independientemente de cuál sea el sexo de la persona. Las carreras académicas de nuestros colegas masculinos no se ven afectadas por la paternidad y las de mis colegas femeninas sí. Entonces no estoy segura de que estemos poniendo en marcha mecanismos que permitan compensar unas responsabilidades y otras." (E20).

Si consideramos la carrera académica, como el sucesivo incremento de méritos profesionales que llevan a obtener figuras contractuales y reconocimiento cada vez superiores, la conclusión es clara: las mujeres obtienen menos méritos y, por lo tanto, tienen menos reconocimiento y visibilidad, obteniendo, además, menos remuneración económica que sus compañeros.

"Somos treinta y tantos [catedráticos] todavía, pero vamos, y subiendo. [...] Con toda seguridad allá donde hay modos patriarcales y estructuras tradicionales muy establecidas es bastante probable que sea muy dificil ser visible siendo mujer, desde luego en mi departamento" (E15).

Los mecanismos de evaluación del profesorado universitario actuales se sustentan en lo meritocrático, sin embargo, una mirada más atenta revela que hay aspectos de la trayectoria vital de una persona que condicionan directamente el acceso a los distintos méritos, siendo así el sistema poco equitativo en realidad. Una de nuestras entrevistadas lo explica claramente:

"Las acreditaciones están lentificando mucho el proceso de acceso a la profesión docente. Incluso hay gente con bastante nivel que huye del sistema porque o no le es rentable o le es muy costoso, es una inversión de muchos años. Y lo que sí es verdad es que puede afectar y conozco algún caso en el que ha afectado el sistema de acreditación al hecho femenino: el coincidir la maternidad con este tipo de procesos puede lentificar" (E7).

Fox (2010) halla resultados similares en su investigación y concluye que los factores que influyen en que las mujeres obtengan menos méritos que los hombres durante su carrera académica van más allá de la maternidad, ya que tanto el trabajo 
como la familia son instituciones que demandan un esfuerzo notable para la mujer y una interfiere en la otra, mientras que para los hombres todavía el trabajo familiar no supone un esfuerzo comparable. Las mujeres reconocen disponer de "menos tiempo", "menos energía" para invertir en su progreso profesional, cuestiones que se traducen en una menor productividad. Este enfoque pone el énfasis en los factores personales del proceso, como la motivación y el capital humano (Toren, 1990), e ignora los factores contextuales, como los estereotipos sexuales o las estructuras organizativas (Hartman, 1987). Otra explicación que se da a la falta de inversión femenina al propio desarrollo profesional es el "doble esfuerzo que el éxito les cuesta a las mujeres científicas", tal como lo demuestran los estudios de García, Fresno y Andreu, (2003). En otras investigaciones realizadas en España se concluye que a los factores anteriormente mencionados se les añaden los estereotipos masculinos y femeninos que se forman con el tiempo. Sánchez García (2011) afirma a partir de sus estudios que los hombres tienen una visión muy estereotipada del género, mientras que las mujeres mantienen posiciones más neutras e igualitarias. Los estereotipos constituyen una realidad que puede estar en la base de comportamientos y actitudes sexistas.

En definitiva, las mujeres lo siguen teniendo más difícil para seguir la carrera académica. Constatar este hecho puede ayudar a entender por qué hay tan pocas mujeres que obtengan cargos de alta responsabilidad en gestión universitaria. Las pocas que 'siguen en la brecha' pasada la época de máxima dedicación a la crianza de los hijos, se dan cuenta de que son prácticamente invisibles porqué durante una época clave no han estado ejerciendo tareas de liderazgo.

"Entiendo que hay una etapa en la vida en que mujeres quieran demostrar este interés [en ocupar cargos de gestión], pero a menudo coincide con esa etapa de la vida en que no es posible [por la maternidad]. [‥] Yo la experiencia que tengo es que la gente que ha participado en gestión, aunque no sea en cargos claves, ganan mucho en visibilidad y son candidatos y candidatas a que después sean representantes, o candidatos a cargos" (E12).

El segundo gran bloque engloba todos los motivos de desigualdad que tienen que ver con la cultura organizativa de las universidades, que ha sido y sigue siendo una institución muy masculinizada. Como hemos visto, lo explican una serie de condiciones culturales determinadas por las cuales las personas académicas han sido durante mucho tiempo casi exclusivamente hombres (Guillamon, 2011). Aunque actualmente hay más mujeres, la universidad ha sido pensada por ellos y su talante es muy masculino. Las profesoras entrevistadas perciben un ambiente bastante hostil, sienten la universidad como una institución "patriarcal o tradicional", que no está muy abierta a la inclusión de las mujeres en sus estructuras y que tiene espacios donde claramente "hay tapones", espacios en los que "está todo tapado".

Parece que la situación sigue pudiéndose diagnosticar en base a la clasificación de Morley (1995), en los que sitúa la universidad y sus departamentos en un continuum en función del estado en que se encuentran en el desarrollo de la igualdad de género: "closed doors" (puertas cerradas), "uneasy environment" (un medio poco fácil), y "open doors" (puertas abiertas). Nuestras entrevistadas perciben claramente algunos contextos universitarios en la fase de "puertas cerradas". 
Un contexto es igualitario cuando favorece la incorporación de las mujeres y se superan las percepciones tradicionales respecto "al rol de la mujer en la división del trabajo, que es una de las causas que genera contextos laborales antipáticos a las mujeres y lo que influye en su deseo de permanecer en el departamento"(Alonso, 2001, 78).

La cultura organizativa masculinizada en la universidad afecta a las profesoras tanto en su actividad investigadora y productiva como al acceso a cargos de cierta responsabilidad y su mantenimiento en ellos. Si quieren acceder y permanecer en un cargo, deben sumergirse en una cultura universitaria que no ha sido creada desde lo femenino y, por consiguiente, en la que puede no haberse contemplado para nada las necesidades y los gustos de ellas, por ejemplo. Una de las personas entrevistadas lo manifiesta así: "Todos sabemos que ha habido una protección hacia el género masculino que no al femenino, entonces hay una tradición" (E10).

Parece suficientemente contrastado el efecto que dicha cultura masculinizada (Rebollo Catalán, García Pérez, Piedra y Vega, 2011) tiene en las profesoras a la hora de incentivar su participación en la universidad. Aquí, se daría lo que García de León (2002) ha llamado "la discriminación de las élites femeninas", ya que las personas que están evaluando la carrera académica son en su mayor parte hombres, porqué no hay mujeres con una categoría suficientemente elevada (catedráticas) para estar en las diversas comisiones de evaluación. A continuación, presentamos una muestra de lo que nuestras participantes dicen al respecto:

"Cuando hice las oposiciones y estaba preparando la cátedra no había ninguna mujer en mi ámbito, yo fui la primera. El proceso de entrada sigue siendo masculino, la toma de decisiones sigue siendo masculina" (E11).

Las investigaciones sobre las élites femeninas evidencian la discriminación de las mujeres. Por un lado, tal como nos comentaba más arriba una de las entrevistadas al recordar su historial profesional, "era la primera [en el ámbito]" y por otro lado se percibe una cierto "malestar" o una realidad "oculta" en relación a los hombres".

Debido a esto se entiende que bastantes profesoras abdiquen de su capacidad para presentarse a procesos de acreditación y a cargos de gestión de rango elevado.

"Y a nivel de arriba, cuando se buscaba vicerrectora era mucho más dificil encontrar vicerrectoras que vicerrectores. En pocas palabras tenías que llamar a 10 mujeres para conseguir una, mientras que llamando a dos hombres conseguias uno" (E1).

La mayoría de profesoras que se dedican a la gestión lo hacen en posiciones de poder menor que las posiciones ocupadas por profesores. El trabajo de Tomás y otros (2010) aporta cifras concluyentes al respecto y se puede afirmar que en nuestro panorama universitario en general hay algunas directoras de departamento, pocas vicedecanas, menos decanas y muchas menos vicerrectoras y rectoras.

Las mujeres que se han querido implicar en profesiones o puestos dominados por los hombres, han encontrado tradicionalmente fuertes resistencias en la cultura de trabajo (García de Léon, 2002) de la cual las mujeres son excluidas (Bagilhole, 1993). A estos estudios se añaden las contribuciones ya clásicas de Schein (1994) y también 
las más recientes de Donoso, Figuera y Rodríguez Moreno (2011) que concluyen que a pesar del aumento numérico de las mujeres en la gestión, el estereotipo de género continua y representa la barrera más importante en el camino de las mujeres, siendo una muestra clara de marginación. Para eliminar esta barrera en el acceso de las mujeres a los puestos de más poder se requiere un proceso de "aculturación" como lo denominan García, Fresno y Andreu (2003) por el que la mujer pase a ser considerada activa y participativa en vez de pasiva.

A pesar de todo lo visto hasta aquí, debemos mencionar que en nuestro estudio hay un sector de personas participantes que no perciben diferencias debidas al género en el acceso y en la promoción académica.

"El acceso a la carrera universitaria, yo creo que es por igual. Hoy día, en pleno siglo XXI, incluso diría que hay más acceso femenino que masculino. La prueba la tenemos en nuestra casa, han entrado más mujeres en la última década que en la anterior" (E7).

Dichas percepciones pueden resultar un tanto inquietantes si se tiene en cuenta el trabajo de Arranz (2004) como se argumenta a continuación. En primer lugar hemos visto la tendencia a considerar que los criterios de evaluación pueden ser 'objetivos' y dejar al margen cuestiones de género que incidan directamente en la igualdad de oportunidades. En segundo lugar se tiende a pensar que el futuro será mejor que el presente, más igualitario y equitativo, en el que las mujeres serán más visibles por sus méritos profesionales y académicos. La autora advierte sobre el caso sueco, alemán u holandés, "donde en un espacio de tiempo que duplica como mínimo al nuestro [español] en el reconocimiento de la igualdad de género, continua manteniéndose semejante el desequilibrio de las cifras entre el profesorado" (2004,3). Es decir, que creer que el tiempo jugará a favor de la equidad entre géneros y que no es necesario tomar medidas para garantizar dicha equidad, parece, cuanto menos, arriesgado.

\section{Consideraciones en torno a las políticas de igualdad en el ámbito universitario estudiado}

Las personas participantes en nuestra investigación comentan su parecer respecto de las políticas de cuotas, por las que se ha de garantizar la presencia de un mínimo de mujeres en diversos órganos de toma de decisiones.

Quien cree que intentar garantizar la paridad es una medida efectiva, alude al incremento numérico real de las profesoras en puestos de poder y con visibilidad.

"Hubo un director, creo que hace cinco o seis años, que se tomó en serio esto [la política de género] y creó una comisión de mujer y ciencia. A partir de ahí llevaron bastante a raja-tabla la normativa o la ley de igualdad y pusieron, en todas las comisiones que actuaban, en los niveles más altos hasta los más bajos, tantas mujeres como hombres" (E15).

La cuota académica, como medida para asegurar el mismo nivel de participación entre hombres y mujeres en la universidad, ha sido analizada por varios estudios. Los autores no la consideran como una estrategia para el cambio deseado, sino como un 
"correctivo contra el mero transcurso del tiempo, que tan poco eficaz se muestra como factor de cambio, y dar una cierta carta de naturaleza a las mujeres en el poder académico", tal y como lo expone García de León $(2002,465)$.

La escasa presencia femenina afecta también a la composición de los tribunales o de los comités de evaluación, contribuyendo a que se perpetúen patrones masculinos entre el examinado y el examinador, un automatismo de autoridad que, en según Bourdieu (2000), recrea la violencia simbólica que tradicionalmente ha acompañado a la masculinidad. Uno de nuestros entrevistados apunta:

"A la hora de elegir una mujer [para garantizar la paridad]... pues, bueno, que te tengo que explicar... determinados catedráticos de aqui jse han puesto! Incluso, el presidente del tribunal dijo que mejor no lo hiciéramos porque si no siempre dirán que esta persona ha entrado por esto, por el género" (E1).

Otros participantes en el estudio insinúan claramente que en cierto grado detectan una "doble moral" en relación a la política de cuotas, consistente en adoptar las medidas consideradas "políticamente correctas" pero sin convicción real.

"Los rectores se han preocupado por aquello del qué dirán, de que haya paridad, pero yo creo que más que nada por la cuestión estética, más que porque estén convencidos" (E17).

"Desde el punto de vista institucional de los documentos escritos, cada vez se intenta ser más políticamente correctos, por tanto, no habrá una discriminación evidente, sino que habrá una discriminación escondida, un 'shadow'” (E4).

Hay participantes, por otro lado, que se manifiestan claramente en desacuerdo con las políticas de paridad adoptadas. Se considera que esta medida de integración puede tener consecuencias indeseables al conducir al uso de criterios, como el género, no estrictamente profesionales y esa idea es compartida tanto por mujeres como por hombres.

"La leyenda de la igualdad me parece absurda, realmente si tienen que ser diez mujeres que sean diez y si no hay mujeres pues que sean diez hombres, ahora si tienen que ser siete y tres, pues siete y tres; eso de cinco y cinco me parece una barbaridad"(E19).

Otra de las medidas adoptadas por las Administraciones Públicas son las ayudas para asegurar que se incluyan mujeres en los proyectos de investigación o que sean ellas las que los dirijan. También hallamos dos posturas diametralmente opuestas en relación a estas ayudas que la Administración Pública promueve, destinadas a impulsar que las mujeres estén al frente de grupos de investigación y presentes en los equipos que se presentan a las convocatorias de proyectos de investigación competitivos. Las personas que se manifiestan contrarias arguyen que está amenazada la "objetividad" en los criterios de evaluación.

"A muchas de las mujeres que colaboran en los proyectos de investigación cuando leen en el BOE que nos van a dar más puntos a los proyectos de investigación si hay mujeres, casi les indigna, aunque sean mujeres les parece mal. [‥] Si tengo que evaluar a 
alguien miro su trayectoria docente, su trayectoria profesional, su trayectoria científica...pero el sexo me da igual...” (E16).

A parte de estas medidas para la igualdad, existen otra serie de estrategias que se aplican desde los departamentos, aunque tienen escasa incidencia por falta de tradición o por no considerarse suficientemente importantes. Una de las entrevistadas apunta:

"Se están haciendo varias cosas como intentar buscar más referencias bibliográficas de trabajos hechos por mujeres, en el sentido de que muchas veces no es porque no haya, sino porque no se buscan, no hay una tradición en este tema" (E1).

Velar por el uso no sexista del lenguaje es otra de estas medidas que se van introduciendo con dificultad en los departamentos universitarios.

"Las mujeres han sido invisibles socialmente, a lo largo de los siglos, en nuestra civilización, occidental [‥] el lenguaje público no estaba adaptado a las mujeres [‥] Ahora resulta que las mujeres sí estamos, desde hace 50 años para acá, por decir mucho. Pues el lenguaje se tiene que adaptar, tiene que evolucionar, y como la evolución del

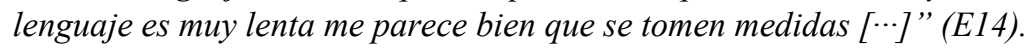

\section{Conclusiones}

El estudio ofrece elementos que contribuyen a comprender, cómo se vive en la universidad actual, el tema de la igualdad de géneros entre el profesorado. Parece que el género sigue siendo un elemento de desigualdad a pesar de las medidas universitarias que se están aplicando. Podríamos considerar que existe una brecha entre lo "oficialmente escrito" y lo "realmente vivido". El estudio muestra que continúa el debate sobre las resistencias al cambio y la dificultad de acompañar las estrategias de mejora institucional con una transformación de la mentalidad androcéntrica tradicional, que sigue siendo existente en muchos ámbitos sociales. Si se quiere la equidad entre géneros, en el campo de la educación superior necesitamos acabar con modelos de reproducción social y optar por modelos transformadores. Hay que seguir trabajando para implementar medidas eficaces tanto a nivel organizativo como a nivel social.

Confiamos en que seguirá estudiándose la cuestión. Creemos que además de estudios de corte cuantitativo que permitan la generalización de los resultados, son necesarios estudios como el que hemos presentado, que analicen en profundidad las percepciones y las creencias de las personas protagonistas de los fenómenos sociales y aporten conocimientos que sí pueden transferirse a contextos similares.

Queremos finalizar afirmando que el tema de la igualdad de género en la universidad sigue suscitando tensiones. Esperamos que nuestro trabajo sirva para invitar a la reflexión y a la toma de decisiones efectiva para una universidad basada en una cultura académica inclusiva e igualitaria. 


\section{Referencias bibliográficas}

ACKER, S. (1996). Doing good and feeling bad: The work of women university teachers. Cambridge Journal of Education, 26, 401-422.

AlBERDI, I. (1996). El poder de las mujeres en las instituciones públicas. En P. Radl, P. (Ed.), Mujeres e institución universitaria en occidente. Conocimiento, investigación y roles de género (69-86). Santiago de Compostela: Universidad de Santiago de Compostela.

Alonso, M.J. (2001). Carrera, trayectoria y estrategias. En: MA. A. GARCIA DE LEON \& M. GARCIA CORTAZAR (Ed.), Las académicas. Profesorado universitario y género (123-154). Madrid: Ministerio de Trabajo y Asuntos Sociales.

ANDREU, S. (2002). La Carrera Académica por género (A propósito de dos investigaciones recientes). Revista Complutense de Educación, 13(1), 13-31.

ARRANZ, F. (2004). Las mujeres y la universidad española: estructuras de dominación y disposiciones feminizadas en el profesorado universitario. Política y Sociedad. 41(2), 223-242.

BAGILHOLE, B. (1993). How to keep a good woman down: an investigation of the role of institutional factors in the process of discrimination against women academics. British Journal of Sociology of Education, 14(3), 261-274.

BAGILHOLE, B. (2002). Challenging equal opportunities: Changing and adapting male hegemony in academia. British Journal of Sociology of Education, 23 (1), 19-33.

BLACKMORE, J. \& SACHS, J. (2001). Women leaders in the restructured and internationalised university. En A. BROOKS \& A. MCKINNON (Eds.), Gender and the restructured university (45-66). Buckingham: Open University Press.

BOURDIEU, P. (2000). La dominación masculina. Barcelona: Anagrama.

BRYMAN, A. (2004). Social Research Methods. New York:Oxford.

Brooks, A. (1997). Academic women. Buckingham: Open University Press.

BRYSON, C. \& BARNES, N. (2000). The casualisation of employment in UK higher education. International Perspectives on Higher Education Research, 1, 183-197.

CHESTERMAN, C. (2002). Women's executive development in Australian higher education. En G. HOWIE \& A. TAUCHERT (Eds.), Gender, teaching and research in higher education: Challenges for the 21st century (143-163). Aldershot: Ashgate

COMMISSION OF THE EUROPEAN COMMUNITIES (1996) Quinto Programa de Acción de la Comisión para la igualdad de oportunidades entre hombres y mujeres. Bruxelles: Comisión Europea

DAVID, M. (2003). Personal and political: Feminisms, sociology and family lives. Stoke-on-Trent: Trentham. 
DE LA REY, C. (2001). Women and management in higher education in South Africa. Paper presented to ACU/IOE Seminar on Managing Gendered Change in Selected Commonwealth Universities, Johannesburg, South Africa, February.

DEEM, R. \& OzGA, J. (2000). Transforming post-compulsory education? Femocrats at work in the academy. Women's Studies International Forum, 23(2), 153-166.

Donoso, T., FiguerA, P. Y RodríGuez MORENO, M. L. (2011). Barreras de género en el desarrollo profesional de la mujer universitaria. Revista de educación, 355, 187212.

Drago, R., Colbeck, C. L., Stauffer, K.D., Pirretti, A., Burkum, K, Fazioli, J., LAZZARO, G., HABASEVICH, T. (2006). The avoidance of bias against caregiving: The case of academic faculty. American Behavioral Scientist, 49, 1222-1247.

EURYDICE (2010). Gender Differences in Educational Outcomes. European Commision.

Evans, P. (2007). Comparative perspectives on changes to Canada's paid parental leave: Implications for class and gender. International Journal of Social Welfare, $16,119-128$.

FOX, M.F. (2010). Women and Men Faculty in Academic Scienceand Engineering: Social-Organizational Indicators and Implications. American Behavioral Scientist, 53(7), 997-1012.

García De LeÓn, M A. (2002). Herederas y heridas. Sobre las élites profesionales femeninas. Madrid: Feminismos.

GArcíA, M.A, Fresno M., M. Y Andreu Mediero, S. (2003). Las investigadoras científicas (Análisis sociológico del campo científico desde la perspectiva de género). Revista Complutense de Educación 14(2), 337-360.

Gault, B. \& Lovell, V. (2006). The costs and benefits of policies to advance work/life integration. American Behavioral Scientist, 49, 1152-1164.

GUILlamon, C. (2011). Los condicionantes de la carrera investigadora en la Universidad que encuentran las mujeres. En: M. TOMAS (coord.) La Universidad vista desde una perspectiva de género (77-112). Barcelona: Octaedro.

HARTMAN, H. (1987). Internal labor markets and gender: a case study of promotion, En C. BROWN \& J. PECHMAN (eds.), Gender in the Workplace (59-105). Washington D.C.: Brookings Institute.

HOWIE, G. \& TAUCHERT, A. (Eds.). (2002). Gender, teaching, and research in higher education: Challenges for the 21st century. Aldershot: Ashgate.

HUSU, LISA. (2000). Gender discrimination in the promised land of gender equality. Higher Education in Europe, XXV(2), 221-228. 
HARLEY, S. (2003). Research Selectivity and Female Academics in UK Universities: from gentleman's club and barrack yard to smart macho. Gender and Education, 15 (4), $377-392$.

KODATE, N; KODATE, K y KODATE, T (2010). Mission Completed? Changing Visibility of Women's Colleges in England and Japan and Their Roles in Promoting Gender Equality in Science. Minerva: A Review of Science, Learning and Policy, 48 (3), 309-330.

LEATHWOOD, C. \& READ, B. (2008) Gender and the changing face of higher education: a feminized future. Buckingham: Open University Press.

LEONARD, D. (2001). A women's guide to doctoral studies. Buckingham: Open University Press.

LuKE, C. (2001). Globalization and women in academia: North/West-South/East. Mahwah, NJ, USA: Lawrence Erlbaum Publishers.

LunN, M. (2007). Women Academicians: Gender and Career Progression. Journal Pendidikan, 32, 77-90

Morales, M. J., LunA, M. J. \& EstebAn, A.I. (2010). Diagnostico de paridad en la universidad: análisis a través de indicadores. Revista de Universidad y Sociedad del conocimiento, 7(2), 1-14

MORLEY, L. (1995). Open Forum an Agenda for Gender Women in the University An Agenda for Gender: Women in the University. European Journal of Women's Studies, 2, 227-275.

MORLEY, L. (2005). Gender equity in Commonwealth higher education. Women's Studies International Forum, 28, 209-221.

Probert, B., EWER, P. \& Whiting, K. (1998). Gender pay equity in Australian higher education. Melbourne: National Tertiary Education Union.

REES, T. (1998). Mainstreaming Equality in the European Union: Education, Training and Labor Market Policies. New York: Routledge.

Rebollo Catalán, Ma A. García Pérez, R., Piedra, J. \& Vega, L. (2011). Diagnóstico de la cultura de género en educación: Actitudes del profesorado hacia la igualdad. Revista de Educación, 355, 503-532.

Sánchez García, M., SuÁrez Ortega, M., Manzano Soto, N., Oliveros Martín-VARÉs, L., LOZANO SANTIAGO, S., FernáNDEZ D' ANDREA, B. Y MALIK LIÉVANO, B. (2011). Estereotipos de género y valores sobre el trabajo entre los estudiantes españoles. Revista de Educación, 355, 331-354.

SCHEIN, V. (1994). Managerial sex typing: A persistent and Persuasive barrier to women's opportunities. En: DAVIDSON, M. \& BURKE, R. (Eds.) Women in Management: Current research Issue (41-52), London: Paul Chapman 
Søyland, A., Skarsbø, A.M., Amble, N., Christensen, L. \& Ølnes, A. (2000). Strategies for Achieving Gender Equality in Higher Education and Research in Norway. Higher Education in Europe, XXV, 2, 147-153

TOMÁs, M., LAVIÉ, J.M., DURAN, M. \& GUILLAMÓN, C. (2010). Women in Academic Administration at the University. Educational Management Aministration and Leadership, 38 (4), 487-498.

Toren, N. (1990) The nexus between family and work roles of women academics: reality and representation. Sex Roles, 24, 651-667.

WoODS, P. (1989). La escuela por dentro. La etnografía en la investigación educativa. Barcelona: Paidós.

\section{Fuentes Electrónicas}

ION, G. \& DURAN, M. (2011) Strengthening women research careers: successful experiences in the Catalan universities. Comunicación presentada a BERA Annual Meeting, Londres, 6-8 septiembre 2011. http://www.leeds.ac.uk/educol/documents/204853.pdf (Consulta: 13/12/ 2011)

OBSERVATORI PER L'IGUALTAT (2011) Jornada La presència de les dones als càrrecs de dirección.: http://www.uab.cat (Consulta: 20/12/2011)

\section{Correspondencia con la autora}

Georgeta ION

Universidad Autónoma de Barcelona

Departamento de Pedagogía Aplicada, G6- 248

Cerdanyola del Valles

Correo-e: georgeta.ion@uab.cat 